\title{
Research Paper: Risk Factors of Hospital-acquired Thrombocytopenia in Toxicological Intensive Care Unit
}

Haleh Talaie $^{1^{*}}$ (D), Sayed Masoud Hosseini² (D, Maryam Nazari' ${ }^{\text {(D), Mehdi Salavati Esfahani³ }}$ Behjat Barari $^{1}$ (D)

1. Toxicological Research Center, Loghman Hakim Hospital, Shahid Beheshti University of Medical Sciences, Tehran, Iran.

2. Departments of Pharmacology and Toxicology, School of Pharmacy, Shahid Beheshti University of Medical Sciences, Tehran, Iran.

3. Department of Toxicology and Pharmacology, Pharmaceutical Sciences Branch, Islamic Azad University, Tehran, Iran.

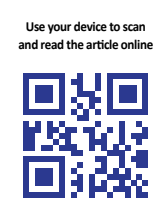

Cftation: Talaie T, Hosseini SM, Nazari M, Salavati Esfahani M, Barari B. Risk Factors of Hospital-acquired Thrombocytopenia in Toxicological Intensive Care Unit International Journal of Medical Toxicology and Forensic Medicine. 2020; 10(3):32256. https://doi.org/10.32598/ijmtfm.v10i3.32256

https://doi.org/10.32598/ijmtfm.v10i3.32256

\section{Article info:}

Received: 14 Sep 2020

First Revision: 23 Sep 2020

Accepted: 04 Nov 2020

Published: 08 Nov 2020

\section{ABSTRACT}

Background: Platelet count is a readily available biomarker predicting disease severity and risk of mortality in the intensive care units (ICU). This study aims to describe the frequency, to assess the risk factors, and to evaluate the impact of thrombocytopenia on patient outcomes in a Toxicological ICU (TICU).

Methods: In this prospective observational Cohort study, we enrolled 184 patients admitted to our TICU from October 1st, 2019, to August 23rd, 2020. Mild/moderate and severe thrombocytopenia were defined as at least one platelet counts less than $150 \times 103 / \mu \mathrm{L}$ and $50 \times 103 / \mu \mathrm{L}$ during the ICU stay, respectively.

Results: Of 184 enrolled patients, $45.7 \%$ had mild to moderate thrombocytopenia and $5.4 \%$ had severe thrombocytopenia. Old age (OR: 1.042, 95\%CI: 1.01-1.075, $\mathrm{P}=0.01$ ), male gender (OR: $4.348,95 \% \mathrm{CI}: 1.33-14.22, \mathrm{P}=0.015)$, increased international normalized ratio (INR) levels (OR: $3.72,95 \% \mathrm{CI}: 1.15-112, \mathrm{P}=0.028$ ), and administration of some medications including heparin (OR: 3.553, 95\%CI: 1.11-11.36, $\mathrm{P}=0.033$ ), antihypertensive drugs (OR: 2.841, 95\%CI: 1.081-7.471, $\mathrm{P}=0.034$ ), linezolid (OR: 13.46, 95\%CI: 4.75-38.13, $\mathrm{P}<0.001$ ), erythromycin (OR: 19.58, 95\% $\mathrm{CI}$ : 3.23-118.86, $\mathrm{P}=0.001$ ), and colistin (OR: 10.29, 95\% CI 1.44-73.69, $\mathrm{P}=0.02$ ) were the risk factors of hospital-acquired thrombocytopenia. The outcomes of patients with normal platelet count were significantly better than those who developed thrombocytopenia $(\mathrm{P}<0.001)$.

Conclusion: We found that thrombocytopenia could develop in almost $50 \%$ of patients admitted to TICU, which is associated with poor prognosis. Additionally, the platelet counts should be closely monitored to administer some medications (heparin, antihypertensive drug, and linezolid), especially in old patients. 


\section{Introduction}

hrombocytopenia, defined as a reduction of platelet counts lower than $\mathbf{T}$ $150000 / \mu \mathrm{L}$, is a concerning issue in the intensive care unit (ICU) [1-5]. Platelet count is a readily available biomarker predicting disease severity and risk of mortality in the ICU $[1,6,7]$. Based on some studies, the frequency of low platelet counts is between $15 \%$ and $58 \%$, which can vary according to thrombocytopenia definition and clinical setting $[3,8,9]$. During ICU stay, developing thrombocytopenia is more associated with prognostic significance than low platelet counts on admission [10].

There have been some mechanisms generally attributed to thrombocytopenia, including decreased bone marrow production, sequestration usually in an enlarged spleen, and increased platelet distraction/consumption [11]. Additionally, this disorder can be precipitated due to some reasons, such as [12] nutritional insufficiency (folate deficiency), human immunodeficiency virus, chemotherapy or cytotoxic medications, infections, endocarditis, chronic liver diseases (cirrhosis), inflammatory diseases, metabolic disorders (hypercalcemia, hyperuricemia), tumor necrosis syndrome, and renal failure. Furthermore, based on Goldfrank's Toxicologic Emergencies and Harrison's Principles of Internal Medicine, some chemicals and medications can decrease the platelet count. These chemicals and medications include alcohol, diazepam, lorazepam, digoxin, valproate, carbamazepine, vancomycin, linezolid, penicillin, ranitidine, acetaminophen, and imipramine $[11,13]$. Some of these medicines are routinely administered in ICUs [14], especially in toxicological ICUs (TICUs), which is a place that many patients are admitted with overdoses of mentioned medicines.

Spontaneous bleeding into vital organs, including the gastrointestinal tract, brain, heart, and adrenal glands, is the primary manifestation of thrombocytopenia, which may necessitate platelet transfusions [15]. Some studies assessed the risk factors of developing thrombocytopenia retrospectively [16-18]. The administration of heparin, vancomycin, nonsteroidal anti-inflammatory drugs before ICU admission, dialysis during the ICU stay, sepsis syndrome/septic shock, liver function abnormalities, and hemodynamic instability are the main risk factors attributed to this homeostatic disorder.
This study aimed to describe the frequency, to assess the risk factors, and to evaluate the impact of thrombocytopenia on patient outcomes in TICU.

\section{Materials and Methods}

\subsection{Study design and participants}

This prospective observational cohort study was conducted in the TICU of Loghman-Hakim Hospital in Tehran, Iran. The studied hospital is a unique tertiary care teaching and referral poison treatment center with an average of 25000 inpatients and outpatients annually. Consecutive patients admitted to the TICU from October 1st, 2019, to August 23rd, 2020, were eligible for the study if they were aged 12 years or older and expected to be in the TICU for more than 72 hours. The exclusion critaria included thrombocytopenia (platelet counts $<150 \times \times$ $103 / \mu \mathrm{L})[17]$ on admission, and also the history of platelet disorders (e.g., idiopathic thrombocytopenic purpura, congenital thrombocytopenia, hypersplenism), hematologic malignancies, use of chemotherapy (in the last 30 days before admission), and mechanical heart valves.

For data analysis, thrombocytopenia was defined as at least one platelet counts less than $150 \times 103 / \mu \mathrm{L}$ and severe thrombocytopenia as at least one platelet counts less than $50 \times 103 / \mu \mathrm{L}$ during the ICU stay [17]. Therefore, 84 thrombocytopenic patients were designated as the case group, and 100 patients with no record of platelet count less than $150 \times 103 / \mu \mathrm{L}$ on admission and during ICU stay were designated as the control group. The informed consent was obtained from the patients or their next of kin. The study was approved by the Ethics Review Committee in Research Deputy Department of Shahid Beheshti University of Medical Sciences, Tehran, Iran (REC Code: IR.SBMU.RETECH.REC.1398.368).

Demographic data (age, sex, underlying disease), type of poisoning, vital signs, glomerular filtration rate (GFR), paraclinical factors, ICU and poison ward length of stay, medications in a period of ICU stay, all complications during ICU stay, and outcomes were recorded for all patients. Lab tests were assessed at baseline, including complete blood count (white blood cells [WBCs], red blood cells [RBCs], hemoglobin $[\mathrm{Hb}]$, hematocrit [Hct], platelets [PLTs]), liver enzymes (aspartate aminotransferase $[\mathrm{AST}]$ and alanine aminotransferase $[\mathrm{ALT}]$ ), sodium, potassium, albumin, bilirubin-total, urea, creatinine $(\mathrm{Cr})$, creatine phosphokinase (CPK), prothrombin time (PT), partial thromboplastin time (PTT), and international normalized ratio (INR). PLTs were also re- 
corded daily throughout the treatment course. GFR was calculated using the Cockroft-Gault formula [19].

\subsection{Statistical analysis}

The statistical analysis was performed in SPSS v. 18, Chicago, Ill, USA). The categorical variables were expressed as frequency (percentage) and numerical variables as Mean \pm SD. The categorical variables were univariately compared using the $\chi^{2}$ test and other quantitative variables using the student's t test. A P value of less than 0.05 was considered statistically significant. Multivariable logistic regression using backward likelihood ratio (LR) selection procedure was used to determine the independent predictors of thrombocytopenia incidence. The dependent variable was thrombocytopenia occurrence (condition of platelet counts less than $150 \times 103 / \mu \mathrm{L}$ during the ICU stay). The independent variables were selected for submission to the model if the univariate $\mathrm{P}$ value was $<0.05$ and had less than $10 \%$ missing data. The following variables were submitted to the model: age, gender, dialysis demand, the incidence of rhabdomyolysis or anuria or oliguria, prescription of heparin, antihypertensive medications, or antibiotic medications (ciprofloxacin, linezolid, meropenem, erythromycin, colistin, levofloxacin), body temperature of patients on admission, level of AST, ALT, potassium, urea, creatinine, CPK, PT, INR, GFR, and duration of ventilation. For the determination of severe thrombocytopenia predictors, we conducted a binary logistic regression. The platelet counts less than $50 \times 103 / \mu \mathrm{L}$ during the ICU stay was the dependent variable, and age, gender, level of INR, and prescription of heparin or linezolid were independent variables. The goodness of fit of the models was assessed using the area under the receiver Operator Characteristic Curve (ROC) calculation and the Hosmer-Lemeshow chi-square statistic.

\section{Results}

\subsection{Baseline characteristic}

One hundred and eighty-four patients (124 men and 60 women with a Mean \pm SD age of $39.43 \pm 16.27$ years) were included in this study. A total of $84(45.7 \%)$ and 10 $(5.4 \%)$ patients precipitated mild/moderate and severe thrombocytopenia during ICU stay, respectively. Univariate analysis revealed a significant difference in some baseline characteristics between patients who developed thrombocytopenia (case group) and those who did not (control group) (Table 1). Patients in the case group were significantly older than patients in the control group (42.012 \pm 16.1 vs $37.26 \pm 16.21$ years, $\mathrm{P}=0.048)$. Among different patients admitted to the Loghman-Hakim Hospital Poison Center, those who were poisoned by methadone, were more likely to be thrombocytopenic $(\mathrm{P}=0.001)$. Besides, methadone poisoning was significantly higher in male patients $(57[46.0 \%]$ in male vs $7\left[11.7 \%\right.$ in female, $\left.\chi^{2}=21, \mathrm{P}<0.001\right)$. Complications such as rhabdomyolysis $(\mathrm{P}=0.017)$, acute tubular necrosis $(\mathrm{P}=0.03)$, pulmonary thromboembolism $(\mathrm{P}=0.007)$, cardiac arrest $(\mathrm{P}=0.29)$, anuria or oliguria $(\mathrm{P}=0.015)$ and dialysis demand $(\mathrm{P}=0.002)$ seemed to be associated with thrombocytopenia. Some of the medications increased the risk of thrombocytopenia. These medications include fentanyl $(\mathrm{P}=0.019)$, norepinephrine $(\mathrm{P}=0.039)$, antihypertensive drugs $(\mathrm{P}=0.049)$, heparin $(\mathrm{P}=0.006)$, erythromycin $(\mathrm{P}=0.012)$, amphotericin $\mathrm{B}(\mathrm{P}=0.002)$, ciprofloxacin $(\mathrm{P}=0.036)$, colistin $(\mathrm{P}=0.002)$, linezolid $(\mathrm{P}<0.001)$, meropenem $(\mathrm{P}=0.031)$, and levofloxacin $(\mathrm{P}=0.007)$. The analysis revealed that concomitant administration of erythromycin and methadone was significantly higher in patients who develop thrombocytopenia than those who did not (8 [88.9\%] vs 1 [11.1\%] respectively, $\chi^{2}=7.1$, $\mathrm{P}=0.008)$. Furthermore, data revealed that body temperature at the time of administration $(\mathrm{P}=0.01)$ and the serum levels of AST $(\mathrm{P}=0.013)$, potassium $(\mathrm{P}=0.008)$, creatinine $(\mathrm{P}=0.002)$, urea $(\mathrm{P}=0.01), \mathrm{CPK}(\mathrm{P}=0.039)$, and the level of PT $(\mathrm{P}=0.002)$, and $\operatorname{INR}(\mathrm{P}=0.002)$ in patients who developed thrombocytopenia were significantly higher than those who did not. The ICU length of stay $(\mathrm{P}=0.021)$, the number of cases needed mechanical ventilation $(\mathrm{P}=0.029)$, and also duration of ventilation $(\mathrm{P}<0.001)$ were higher in patients who developed thrombocytopenia compared to patients with normal platelet count.

\subsection{Outcome}

The patients in the control group had more favorable outcomes than those who suffered from thrombocytopenia (Table 1). The rate of death was significantly higher in patients who developed severe or mild to moderate thrombocytopenia compared to those who did not $(\mathrm{P}<0.001)$, whereas the number of cured patients in the control group was significantly higher than thrombocytopenic patients $(\mathrm{P}<0.001)$.

\subsection{Multivariable analysis}

The multivariable logistic regression model for mild to moderate and severe thrombocytopenia occurrence is depicted in Table 2. The independent predictors of mild to moderate thrombocytopenia were age (OR: 1.042, 95\%CI: 1.01-1.075, $\mathrm{P}=0.01)$, gender (OR: 4.348, 95\%CI: $1.33-14.22, \mathrm{P}=0.015)$, level of INR at the time 
Table 1. Univariate analysis of baseline characteristics of 184 patients

\begin{tabular}{|c|c|c|c|c|c|}
\hline \multirow{2}{*}{\multicolumn{2}{|c|}{ Demographics }} & \multicolumn{3}{|c|}{ No. (\%)/ Mean \pm SD } & \multirow{2}{*}{$\mathbf{P}$} \\
\hline & & Control $(n=100)$ & Case $(n=84)$ & Statistic/ $\chi^{2} / \mathrm{t}$ & \\
\hline & Age $(y)$ & $37.26 \pm 16.21$ & $42.012 \pm 16.1$ & $t=1.99$ & 0.048 \\
\hline \multirow[b]{2}{*}{ Gender, } & Male & $58(46.8)$ & $66(53.2)$ & \multirow[b]{2}{*}{$\chi^{2}=8.8$} & \multirow[b]{2}{*}{0.003} \\
\hline & Female & & & & \\
\hline \multirow{7}{*}{ Poisoning } & Benzodiazepine & $52(61.9)$ & $32(38.1)$ & 3.5 & 0.059 \\
\hline & Methadone & $24(37.5)$ & $40(62.5)$ & 11.23 & 0.001 \\
\hline & Tramadol & $10(55.6)$ & $8(44.4)$ & 0.012 & 0.914 \\
\hline & Amphetamine & $10(52.6)$ & $9(47.4)$ & 0.025 & 0.874 \\
\hline & Aluminum phosphide & $7(46.7)$ & $8(53.3)$ & 0.388 & 0.533 \\
\hline & Opiate & $18(54.5)$ & $15(45.5)$ & 0.001 & 0.98 \\
\hline & Tricyclic antidepressant & $5(71.4)$ & $2(28.6)$ & 0.856 & 0.355 \\
\hline \multirow{5}{*}{ Medication history } & Heart medications & $4(30.8)$ & $9(69.2)$ & 6.3 & 0.043 \\
\hline & Pulmonary medications & $0(0)$ & $2(100)$ & 11.06 & 0.004 \\
\hline & Neurological medications & $8(34.8)$ & $15(65.2)$ & 7.07 & 0.029 \\
\hline & Gastrointestinal medications & $0(0)$ & $1(100)$ & 3.076 & 0.215 \\
\hline & Sedative medications & $4(36.4)$ & $7(63.6)$ & 9.99 & 0.007 \\
\hline \multirow{11}{*}{$\begin{array}{l}\text { Complications } \\
\text { during ICU stay }\end{array}$} & Sepsis & $5(38.5)$ & $8(61.5)$ & 1.4 & 0.233 \\
\hline & Rhabdomyolysis & $18(39.1)$ & $28(60.9)$ & 5.72 & 0.017 \\
\hline & Acute tubular necrosis (ATN) & $1(14.3)$ & $6(85.7)$ & 4.71 & 0.03 \\
\hline & Deep vein thrombosis (DVT) & $3(30)$ & $7(70)$ & 2.53 & 0.112 \\
\hline & Pulmonary thromboembolism & $0(0)$ & $6(100)$ & 7.38 & 0.007 \\
\hline & $\begin{array}{l}\text { Disseminated intravascular } \\
\text { coagulation (DIC) }\end{array}$ & $2(66.7)$ & $1(33.3)$ & 0.187 & 0.67 \\
\hline & Multiple organ failure (MOF) & $1(33.3)$ & $2(66.7)$ & 0.543 & 0.461 \\
\hline & Cardiac arrest & $9(34.6)$ & $17(65.4)$ & 4.75 & 0.029 \\
\hline & $\begin{array}{l}\text { Acute respiratory distress } \\
\text { syndrome }\end{array}$ & $9(52.9)$ & $8(47.1)$ & 0.015 & 0.903 \\
\hline & Anuria or oliguria & $14(36.8)$ & $24(63.2)$ & 5.915 & 0.015 \\
\hline & Dialysis & $9(29)$ & $22(71)$ & 9.63 & 0.002 \\
\hline \multirow{5}{*}{$\begin{array}{l}\text { Medications } \\
\text { during ICU stay }\end{array}$} & Midazolam & $80(51.6)$ & $75(48.4)$ & 2.5 & 0.112 \\
\hline & Acetaminophen & $21(53.8)$ & $18(46.2)$ & 0.005 & 0.944 \\
\hline & Gabapentin & $0(0)$ & $3(100)$ & 3.63 & 0.057 \\
\hline & Vitamin B6 & $7(23.3)$ & $23(76.7)$ & 13.9 & $<0.001$ \\
\hline & Vitamin K & $4(66.7)$ & $2(33.3)$ & 0.38 & 0.54 \\
\hline
\end{tabular}




\begin{tabular}{|c|c|c|c|c|c|}
\hline & \multirow{2}{*}{ Demographics } & \multicolumn{3}{|c|}{ No. (\%)/ Mean \pm SD } & \multirow{2}{*}{$\mathbf{P}$} \\
\hline & & Control $(n=100)$ & Case $(n=84)$ & Statistic/ $\chi^{2} / t$ & \\
\hline & Folic acid & $4(66.7)$ & $2(33.3)$ & 0.38 & 0.54 \\
\hline & Pulmonary medications & $22(66.7)$ & $11(33.3)$ & 2.46 & 0.117 \\
\hline & Diabetic medications & $12(66.7)$ & $6(33.3)$ & 1.22 & 0.27 \\
\hline & Ranitidine & $70(50.7)$ & $68(49.3)$ & 2.92 & 0.087 \\
\hline & Pantoprazole & $41(51.2)$ & $39(48.8)$ & 0.547 & 0.459 \\
\hline & Anti-vomiting & $31(50)$ & $31(50)$ & 0.712 & 0.399 \\
\hline & Fentanyl & $76(50.3)$ & 75 (49.7) & 5.47 & 0.019 \\
\hline & Methadone & $35(47.3)$ & 39 (52.7) & 2.48 & 0.115 \\
\hline & Norepinephrine (NEP) & $16(40)$ & $24(60)$ & 4.24 & 0.039 \\
\hline & Furosemide & $14(41.2)$ & $20(58.8)$ & 2.92 & 0.088 \\
\hline & $\begin{array}{c}\text { Antihypertensive medications, } \\
\text { No. (\%) }\end{array}$ & $30(44.8)$ & $37(55.2)$ & 3.9 & 0.049 \\
\hline & Clopidogrel & $1(25)$ & $3(75)$ & 1.42 & 0.233 \\
\hline & Heparin & $67(48.6)$ & $71(51.4)$ & 7.48 & 0.006 \\
\hline & Aspirin & $8(66.7)$ & $4(33.3)$ & 0.758 & 0.376 \\
\hline & Amikacin & $14(45.2)$ & $17(54.8)$ & 1.27 & 0.26 \\
\hline & Erythromycin & $6(28.6)$ & $15(71.4)$ & 6.35 & 0.012 \\
\hline \multirow[t]{16}{*}{$\begin{array}{l}\text { Medications } \\
\text { during ICU stay }\end{array}$} & Acyclovir & $1(20)$ & $4(80)$ & 2.44 & 0.118 \\
\hline & Ampicillin-Sulbactam & $9(52.9)$ & $8(47.1)$ & 0.015 & 0.903 \\
\hline & Piperacillin-Tazobactam & $7(36.8)$ & $12(63.2)$ & 2.62 & 0.106 \\
\hline & Amphotericin B & $1(9.1)$ & $10(90.9)$ & 9.5 & 0.002 \\
\hline & Cefazolin & $2(66.7)$ & $1(33.3)$ & 0.187 & 0.66 \\
\hline & Ceftazidime & $5(35.7)$ & $9(64.3)$ & 2.12 & 0.145 \\
\hline & Ciprofloxacin & $27(43.5)$ & $35(56.5)$ & 4.4 & 0.036 \\
\hline & Ceftriaxone & $45(50)$ & $45(50)$ & 1.34 & 0.247 \\
\hline & Clindamycin & $49(51)$ & $47(49)$ & 0.884 & 0.347 \\
\hline & Colistin & $8(33.3)$ & $16(66.7)$ & 4.91 & 0.027 \\
\hline & Linezolid & $15(22.1)$ & $53(77.9)$ & 45.32 & $<0.001$ \\
\hline & Meropenem & 46 (46.9) & $52(53.1)$ & 4.64 & 0.031 \\
\hline & Imipenem & $18(72)$ & $7(28)$ & 3.63 & 0.057 \\
\hline & Teicoplanin & $4(36.4)$ & $7(63.6)$ & 1.52 & 0.217 \\
\hline & Levofloxacin & $4(23.5)$ & $13(76.5)$ & 7.17 & 0.007 \\
\hline & Vancomycin & $45(60)$ & $30(40)$ & 1.63 & 0.202 \\
\hline
\end{tabular}




\begin{tabular}{|c|c|c|c|c|c|}
\hline \multirow{2}{*}{\multicolumn{2}{|c|}{ Demographics }} & \multicolumn{3}{|c|}{ No. (\%)/ Mean $\pm S D$} & \multirow{2}{*}{$\mathbf{P}$} \\
\hline & & Control $(n=100)$ & Case $(n=84)$ & Statistic/ $\chi^{2} / t$ & \\
\hline \multirow{6}{*}{$\begin{array}{l}\text { Medications } \\
\text { during ICU stay }\end{array}$} & Gentamycin & $4(80)$ & $1(20)$ & 1.36 & 0.243 \\
\hline & Valproate & $7(87.5)$ & $1(12.5)$ & 3.7 & 0.054 \\
\hline & Dexamethazone & $31(45.6)$ & $37(54.4)$ & 3.34 & 0.068 \\
\hline & Haloperidol & $13(72.2)$ & $5(27.8)$ & 2.57 & 0.109 \\
\hline & Tranexamic acid & $0(0)$ & $2(100)$ & 2.407 & 0.121 \\
\hline & Amiodaron & $1(33.3)$ & $2(66.7)$ & 0.543 & 0.461 \\
\hline \multirow{5}{*}{ Vital sign (range) } & Temperature $\left({ }^{\circ} \mathrm{C}\right)$ & $37.06 \pm 0.388$ & $37.24 \pm 0.532$ & $\mathrm{t}=2.6$ & 0.01 \\
\hline & Heart rate $(/ \mathrm{min})$ & $93.14 \pm 18.1$ & $93.96 \pm 20.2$ & $t=0.292$ & 0.771 \\
\hline & Respiratory rate (/min) & $16.45 \pm 4.2$ & $15.71 \pm 5.3$ & $t=1.05$ & 0.294 \\
\hline & Systolic pressure (mm Hg) & $114.01 \pm 21.36$ & $114.47 \pm 25.3$ & $t=0.134$ & 0.89 \\
\hline & Diastolic pressure $(\mathrm{mm} \mathrm{Hg})$ & $70.49 \pm 14.04$ & $69.36 \pm 16.94$ & $\mathrm{t}=0.496$ & 0.62 \\
\hline \multirow{19}{*}{ Paraclinical factor (range) } & Oxygen saturation (\%) & $93.94 \pm 5.9$ & $93.4 \pm 7.2$ & $t=0.561$ & 0.575 \\
\hline & $\mathrm{WBC}(\times(\times 103 / \mu \mathrm{L})$ & $11.65 \pm 4.9$ & $12.13 \pm 6.1$ & $t=0.36$ & 0.71 \\
\hline & $\operatorname{RBC}(\times(\times 106 / \mu \mathrm{L})$ & $4.78 \pm 0.73$ & $4.77 \pm 0.76$ & $t=0.037$ & 0.971 \\
\hline & $\mathrm{Hb}(\mathrm{g} / \mathrm{dL})$ & $14.01 \pm 2.57$ & $14.48 \pm 2.53$ & $t=1.23$ & 0.22 \\
\hline & Hct (\%) & $42.093 \pm 6$ & $42.77 \pm 6.9$ & $t=0.711$ & 0.48 \\
\hline & $\operatorname{PLT}(\times(\times 103 / \mu \mathrm{L})$ & $83.66 \pm 230.36$ & $295.05 \pm 732.47$ & $t=2.54$ & 0.013 \\
\hline & AST (U/L) & $70.25 \pm 197.66$ & $612.42 \pm 2542.7$ & $t=1.95$ & 0.055 \\
\hline & $\operatorname{ALT}(U / L)$ & $137.83 \pm 3.7$ & $138.2 \pm 3.5$ & $t=0.695$ & 0.488 \\
\hline & Sodium (mEq/L) & $4.05 \pm 0.56$ & $4.35 \pm 0.87$ & $t=2.7$ & 0.008 \\
\hline & Albumin b (g/dL) & $3.82 \pm 0.55$ & $3.63 \pm 0.65$ & $t=1.36$ & 0.177 \\
\hline & Bilirubin-Total (mg/dL) & $1.06 \pm 0.8$ & $1.23 \pm 1.31$ & $t=0.94$ & 0.35 \\
\hline & Creatinine (mg/dL) & $1.25 \pm 0.54$ & $1.65 \pm 1.03$ & $\mathrm{t}=3.2$ & 0.002 \\
\hline & Urea (mg/dL) & $39.32 \pm 25.57$ & $52.44 \pm 39.37$ & $t=2.62$ & 0.01 \\
\hline & Creatine phosphokinase (U/L) & $1681.97 \pm 5099.02$ & $3817.4 \pm 7823.1$ & $t=2.09$ & 0.039 \\
\hline & Alkaline phosphatase (U/L) & $206.16 \pm 109.93$ & $193.27 \pm 55.17$ & $t=1.004$ & 0.317 \\
\hline & Prothrombin time (s) & $13.45 \pm 3.01$ & $14.83 \pm 2.7$ & $t=3.135$ & 0.002 \\
\hline & Partial thromboplastin time (s) & $32.28 \pm 9.74$ & $34.41 \pm 11.83$ & $t=1.3$ & 0.198 \\
\hline & International normalized ratio & $1.19 \pm 0.37$ & $1.44 \pm 0.63$ & $t=3.17$ & 0.002 \\
\hline & ICU length of stay, Mean $\pm S D, d$ & $14.83 \pm 12.39$ & $19.44 \pm 14.12$ & $t=2.33$ & 0.021 \\
\hline
\end{tabular}




\begin{tabular}{|c|c|c|c|c|c|}
\hline \multirow{2}{*}{\multicolumn{2}{|c|}{ Demographics }} & \multicolumn{3}{|c|}{ No. (\%)/ Mean \pm SD } & \multirow{2}{*}{$\mathbf{P}$} \\
\hline & & Control $(n=100)$ & Case $(n=84)$ & Statistic/ $\chi^{2} / t$ & \\
\hline \multirow{5}{*}{ Paraclinical factor (range) } & $\begin{array}{l}\text { Poison Ward length of stay, } \\
\text { Mean } \pm S D, d\end{array}$ & $2.68 \pm 3.54$ & $2.89 \pm 7.62$ & $t=0.249$ & 0.803 \\
\hline & Duration to admit, Mean $\pm S D, d$ & $7 \pm 5.6$ & $11.29 \pm 16.1$ & $\mathrm{t}=1.3$ & 0.2 \\
\hline & Mechanical ventilation, No. (\%) & $68(44.7)$ & $84(55.3)$ & $\chi^{2}=4.8$ & 0.029 \\
\hline & $\begin{array}{l}\text { Duration of ventilation, } \\
\text { Mean } \pm S D, d\end{array}$ & $10.86 \pm 12.87$ & $18.38 \pm 14.5$ & $t=3.65$ & $<0.001$ \\
\hline & $\mathrm{GFR}$, Mean $\pm S D, \mathrm{~mL} / \mathrm{min}$ & $74.64 \pm 23.75$ & $65.13 \pm 29.53$ & $t=2.37$ & 0.019 \\
\hline \multirow{3}{*}{$\begin{array}{l}\text { Mild to moderate throm- } \\
\text { bocytopenic patients' } \\
\text { outcome }\end{array}$} & Cure & $83(83)$ & $42(50)$ & 22.82 & $<0.001$ \\
\hline & Cure with sequel & $2(2)$ & $5(6)$ & 1.95 & 0.163 \\
\hline & Death & $15(15)$ & $37(44)$ & 18.99 & $<0.001$ \\
\hline \multirow{3}{*}{$\begin{array}{l}\text { Severe thrombocytopenic } \\
\text { patients' outcome }\end{array}$} & Cure & $124(71.3)$ & $1(10)$ & 16.29 & $<0.001$ \\
\hline & Cure with sequel & $7(4)$ & $0(0)$ & 0.42 & 0.52 \\
\hline & Death & $43(24.7)$ & $9(90)$ & 19.88 & $<0.001$ \\
\hline
\end{tabular}

of admission (OR: 3.72, 95\%CI: 1.15-112, $\mathrm{P}=0.028$ ), and prescription of some medicines, including linezolid (OR: 13.46, 95\%CI: 4.75-38.13, $\mathrm{P}<0.001$ ), heparin (OR: 3.553, 95\%CI: 1.11-11.36, $\mathrm{P}=0.033$ ), erythromycin (OR: $19.58,95 \% \mathrm{CI}$ : 3.23-118.86, $\mathrm{P}=0.001$ ), colistin (OR: 10.29, 95\%CI: 1.44-73.69, $\mathrm{P}=0.02$ ), and antihypertensive medications (OR: 2.841, 95\%CI: 1.081-7.471, $\mathrm{P}=0.034)$. The independent predictors of severe thrombocytopenia were age (OR: 1.04, 95\%CI: 1.002-1.078,
$\mathrm{P}=0.036$ ) and prescription of linezolid (OR: 4.19, 95\%CI: 1.022-17.15, $\mathrm{P}=0.047$ ).

The goodness of fit of the models was assessed using the area under the receiver ROC calculation and the Hosmer-Lemeshow chi-square statistic. Both regression models for mild/moderate and severe thrombocytopenia fitted well. The area under the ROC for mild/moderate thrombocytopenia model was 0.89 (95\%CI: 0.844 $0.936, \mathrm{P}<0.001$ ), and for severe thrombocytopenia mod-

Table 2. Multivariable logistic regression model for hospital-acquired mild/moderate and severe thrombocytopenia

\begin{tabular}{|c|c|c|c|c|c|c|c|}
\hline \multirow{2}{*}{ Independent Variables } & \multicolumn{3}{|c|}{$<150000 / \mathrm{mm}^{3}$ (mild to moderate) } & \multirow{2}{*}{$\begin{array}{c}\text { Independent } \\
\text { Variables }\end{array}$} & \multicolumn{3}{|c|}{$<50000 / \mathrm{mm}^{3}$ (Severe) } \\
\hline & OR & $95 \% \mathrm{Cl}$ & $\mathbf{P}$ & & OR & $95 \% \mathrm{Cl}$ & $\mathbf{P}$ \\
\hline Age & 1.042 & $1.01-1.075$ & 0.01 & Age & 1.04 & $1.002-1.078$ & 0.036 \\
\hline Gender & 4.348 & $1.33-14.22$ & 0.015 & Linezolid & 4.19 & $1.022-17.15$ & 0.047 \\
\hline International normalized ratio. admit & 3.72 & $1.15-12$ & 0.028 & - & - & - & - \\
\hline Antihypertensive medication & 2.841 & $1.081-7.471$ & 0.034 & - & - & - & - \\
\hline Heparin & 3.553 & $1.11-11.36$ & 0.033 & - & - & - & - \\
\hline Erythromycin & 19.58 & $3.23-118.86$ & 0.001 & - & - & - & - \\
\hline Colistin & 10.29 & $1.44-73.69$ & 0.02 & - & - & - & - \\
\hline Linezolid & 13.46 & $4.75-38.13$ & $<0.001$ & - & - & - & - \\
\hline
\end{tabular}




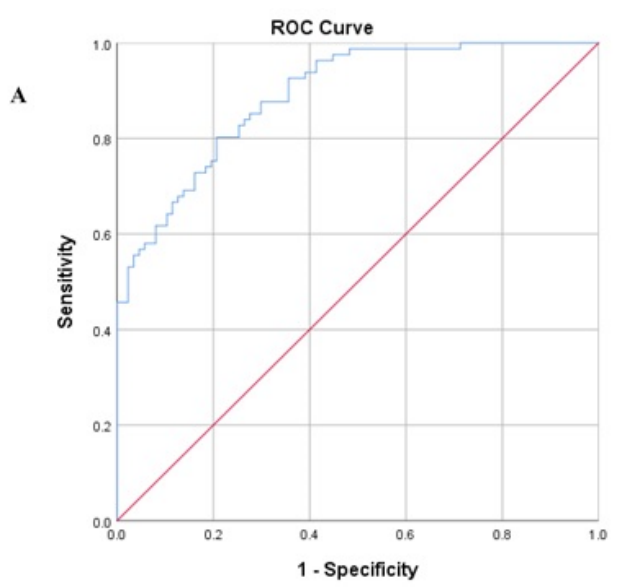

Figure 1. Receiver-operator characteristic (ROC)

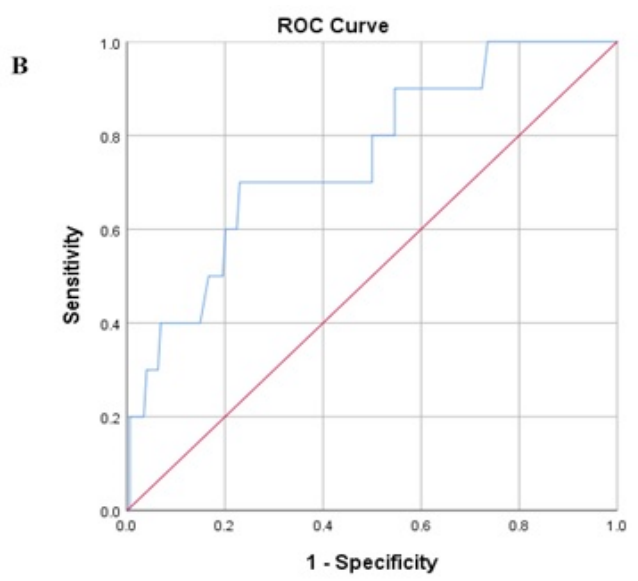

International Journal of
Medical Toxicolbgy \& forensic Medicine

Curve for the multivariable logistic regression model for the prediction of (A) hospital-acquired mild/moderate thrombocytopenia and (B) hospital-acquired severe thrombocytopenia.

el was 0.753 (95\%CI: 0.598-0.908, $\mathrm{P}=0.007)$ (Figure 1). The Hosmer-Lemeshow chi-square statistic for the mild/ moderate thrombocytopenia model was $5.11(\mathrm{P}=0.745)$, and for the severe thrombocytopenia model was 9.31 $(\mathrm{P}=0.317)$.

\section{Discussion}

Thrombocytopenia, as the most prevalent hematological disorder in ICU, is a well-known life-threatening complication that has a substantial prognostic significance related to the patient's outcome [3, 8, 9, 14]. Based on some studies, the prevalence of thrombocytopenia was reported to be $15 \%-85 \%$ during the ICU stay depending on the study population, which was consistent with our findings. Different poisons, including alcohol, opioid, sedative-hypnotic drugs, heavy metals, aluminum phosphide, and also the prescription of some medications like antibiotics (including linezolid, vancomycin, $\beta$-Lactams, rifampin, fluoroquinolones) during TICU stay might result in thrombocytopenia [20-26]. According to our multivariate model, age, gender, INR levels, administration of heparin, antihypertensive medications, and some antibiotics, including linezolid, erythromycin, and colistin, were the main predictors of mild to moderate thrombocytopenia. Furthermore, age and linezolid prescription were the only factors that increased the risk of severe thrombocytopenia.

The platelet count was relatively stable in middle-aged people, whereas it might drop in older people [27]. It is well documented that aging affects platelet count and function, which originate from the change in the hema- topoietic tissue, vascular health, and blood composition. Thrombocytopenia was more prevalent in male patients. The heterogeneity in the cause of poisoning between men and women might explain this finding. For instance, methadone poisoning was significantly higher in male patients, and thrombocytopenia was more prevalent in patients with methadone poisoning. It was similar to previous studies, which indicated that methadone might result in thrombocytopenia, especially in old age men [28]

Antihypertensive drugs like thiazide diuretics and angiotensin receptor blockers might induce thrombocytopenia through the reduction of the Renin-angiotensin System (RAS) activation, myelosuppression, and the acceleration of immune-mediated platelet destruction [29, 30].

Heparin-induced Thrombocytopenia (HIT) is an immune system response in which the platelet factor 4 (PF4) forms an immune complex with heparin and immunoglobulin $\mathrm{G}$ ( $\mathrm{IgG})$. The formed immune complex binds to the platelet surface receptors which results in platelet destructions and thrombocytopenia [31, 32]. Besides, heparin could result in elevated INR levels [33] as it was observed in thrombocytopenic patients (case group).

It was suggested that antibiotics lead to Drug-induced Thrombocytopenia (DIT) through the direct myelosuppression and immune-mediated platelet destruction [26]. As we observed in this study, linezolid and colistin have the potential to induce thrombocytopenia [26, 34]. It was frequently reported that the oxazolidinones, e.g., linezolid, may result in peripheral destruction of thrombocytes. Baseline platelet count, creatinine clearance, and end- 
stage renal disease are the main risk factors of linezolidinduced thrombocytopenia [35-37]. In a case report, the thrombocytopenia was manifested in a 5-year-old female following a colistin prescription. After exclusion of all other probable causes, it was revealed that the colistin was the causative factor for thrombocytopenia development. Furthermore, the levels of thrombocytes returned to normal following the discontinuation of colistin [34].

Thrombocytopenia is not determined as an adverse effect of erythromycin; however, this drug is a cytochrome P450 3A4 inhibitor which could interact with some medications that are cytochrome substrates [38] and thrombocytopenia is their adverse effects like methadone $[39,40]$. Similar to previous studies, we showed that the concomitant administration of erythromycin and methadone (a medication with thrombocytopenia induction potential) [28] was significantly higher in patients who develop thrombocytopenia than those who did not.

Previous studies showed that thrombocytopenia occurrence during ICU stay was accompanied by poor prognosis [4, 41]. Strauss R. et al. (2002) reported that the risk of bleeding in mild to moderate thrombocytopenia was three times and in severe thrombocytopenia was six times higher than those with normal counts of platelets [4]. In this study, the data revealed that the outcomes of patients with hospital-acquired thrombocytopenia were poor. Both mild/moderate and severe thrombocytopenia were significantly associated with increased mortality rates.

\section{Conclusion}

In conclusion, the frequency of mild/moderate and severe thrombocytopenia was $45.7 \%$ and $5.4 \%$, respectively. The main risk factors of thrombocytopenia in TICU were old age, male gender, increased INR levels at the time of admission, administration of heparin, antihypertensive medications, and antibiotics, including linezolid, erythromycin, and colistin. Moreover, the main factors which may predict severe thrombocytopenia are old age and linezolid administration. Therefore, the platelet counts should be monitored closely to administer the mentioned medications, especially in old patients. Further prospective studies with large sample sizes are needed to conduct more detailed investigations about the factors that predict the development of thrombocytopenia in TICU.

\section{Ethical Considerations}

\section{Compliance with ethical guidelines}

The study protocol was approved by the Ethics Review Committee in Research Deputy Department of Shahid Beheshti University of Medical Sciences, Tehran, Iran (REC Code: IR.SBMU.RETECH.REC.1398.368).

\section{Funding}

This research did not receive any specific grant from funding agencies in the public, commercial, or non-profit sectors.

\section{Author's contributions}

All authors were equally contributed in preparing this article.

\section{Conflict of interest}

The authors declared no conflict of interest.

\section{Acknowledgements}

We acknowledge the Toxicological Research Center, Loghman Hakim Hospital, Shahid Beheshti University of Medical Sciences for their support.

\section{References}

[1] Hui P, Cook DJ, Lim W, Fraser GA, Arnold DM. The frequency and clinical significance of thrombocytopenia complicating critical illness: A systematic review. Chest. 2011; 139(2):271-8. [DOI:10.1378/chest.10-2243] [PMID]

[2] Moreau D, Timsit JF, Vesin A, Garrouste-Orgeas M, de Lassence A, Zahar JR, et al. Platelet count decline: An early prognostic marker in critically ill patients with prolonged ICU stays. Chest. 2007; 131(6):1735-41. [DOI:10.1378/ chest.06-2233] [PMID]

[3] Vanderschueren S, De Weerdt A, Malbrain M, Vankersschaever D, Frans E, Wilmer A, et al. Thrombocytopenia and prognosis in intensive care. Critical Care Medicine 2000; 28(6):1871-6. [DOI:10.1097/00003246-200006000-00031] [PMID]

[4] Strauss R, Wehler M, Mehler K, Kreutzer D, Koebnick C, Hahn EG. Thrombocytopenia in patients in the medical intensive care unit: Bleeding prevalence, transfusion requirements, and outcome. Critical Care Medicine. 2002; 30(8):1765-71. [DOI:10.1097/00003246-200208000-00015] [PMID]

[5] Venkata C, Kashyap R, Farmer JC, Afessa B. Thrombocytopenia in adult patients with sepsis: Incidence, risk factors, 
and its association with clinical outcome. Journal of Intensive Care. 2013; 1(1):9. [DOI:10.1186/2052-0492-1-9] [PMID] [PMCID]

[6] Khurana D, Deoke SA. Thrombocytopenia in critically Ill patients: clinical and laboratorial behavior and its correlation with short-term outcome during hospitalization. Indian Journal Of Critical Care Medicine. 2017; 21(12):861. [DOI:10.4103/ ijccm.IJCCM_279_17] [PMID] [PMCID]

[7] Lippi G, Plebani M, Henry BM. Thrombocytopenia is associated with severe coronavirus disease 2019 (COVID-19) infections: A meta-analysis. Clinica Chimica Acta. 2020; 506:145-8. [DOI:10.1016/j.cca.2020.03.022] [PMID] [PMCID]

[8] Kinasewitz GT, et al., Universal changes in biomarkers of coagulation and inflammation occur in patients with severe sepsis, regardless of causative micro-organism [ISRCTN74215569]. Critical Care. 2004; 8(2):R82-90. [DOI:10.1186/cc2459] [PMID] [PMCID]

[9] Stéphan F, Hollande J, Richard O, Cheffi A, Maier-Redelsperger M, Flahault A. Thrombocytopenia in a surgical ICU. Chest. 1999; 115(5):1363-70. [DOI:10.1378/chest.115.5.1363] [PMID]

[10] Nijsten MW, ten Duis HJ, Zijlstra JG, Porte RJ, Zwaveling $\mathrm{JH}$, Paling JC, et al. Blunted rise in platelet count in critically ill patients is associated with worse outcome. Critical Care Medicine. 2000; 28(12):3843-6. [DOI:10.1097/00003246-20001200000017] [PMID]

[11] Jameson JL, Fauci AS, Kasper DL, Hauser SL, Longo DL, Loscalzo J. Harrison's principles of internal medicine. $20^{\text {th }}$ ed. New York: McGraw-Hill Education; 2018.

[12] Arnold DM, Cuker A. Approach to the adult with unexplained thrombocytopenia [Internet]. [Updated: July 2020]. Available from: https://www.lib.utdo.ir/contents/approach-to-the-adult-with-unexplained-thrombocytopenia/pr int?search=thrombocutopenia\&source=search_result\&select edTitle=1 150\&usage_type=default\&display_rank $=1$.

[13] Hoffman RS, Howland MA, Lewin N, Nelson L, Goldfrank L. Goldfrank's Toxicologic Emergencies. $10^{\text {th }}$ ed. New York: McGraw-Hill Education; 2014

[14] Baughman RP, Lower EE, Flessa HC, Tollerud DJ. Thrombocytopenia in the intensive care unit. Chest. 1993; 104(4):1243-7. [DOI:10.1378/chest.104.4.1243] [PMID]

[15] Hine LK, Gerstman BB, Wise RP, Tsong Y. Mortality resulting from blood dyscrasias in the United States, 1984. The American Journal of Medicine. 1990. 88(2):151-3. [DOI:10.1016/0002-9343(90)90465-P]

[16] Bonfiglio MF, Traeger SM, Kier KL, Martin BR, Hulisz DT, Verbeck SR. Thrombocytopenia in intensive care patients: A comprehensive analysis of risk factors in 314 patients. The Annals of Pharmacotherapy. 1995; 29(9):835-42. [DOI:10.117 7/106002809502900901] [PMID]

[17] Crowther MA, Cook DJ, Meade MO, Griffith LE, Guyatt $\mathrm{GH}$, Arnold DM, et al. Thrombocytopenia in medical-surgical critically ill patients: prevalence, incidence, and risk factors. Journal of Critical Care. 2005; 20(4):348-53. [DOI:10.1016/j. jcrc.2005.09.008] [PMID]

[18] Fountain EM, Arepally GM. Etiology and complications of thrombocytopenia in hospitalized medical patients. Journal of Thromb Thrombolysis. 2017; 43(4):429-36. [DOI:10.1007/ s11239-016-1467-8] [PMID]

[19] Hanai Y, Matsuo K, Ogawa M, Higashi A, Kimura I, Hirayama $\mathrm{S}$, et al. A retrospective study of the risk factors for linezolid-induced thrombocytopenia and anemia. Journal of Infection and Chemotherapy. 2016; 22(8):536-42. [DOI:10.1016/j.jiac.2016.05.003] [PMID]

[20] Silczuk A, Habrat B. Alcohol-induced thrombocytopenia: Current review. Alcohol. 2020; 86:9-16. [DOI:10.1016/j.alcohol.2020.02.166] [PMID]

[21] Andersen GO, Ritland S. Life threatening intoxication with sodium valproate. Journal of Toxicology: Clinical Toxicology. 1995; 33(3):279-84. [DOI:10.3109/15563659509018000] [PMID]

[22] Gafter, U., et al., Thrombocytopenia, eosinophilia, and ranitidine. Annals of internal medicine, 1987. 106(3): p. 477-477. [DOI:10.7326/0003-4819-106-3-477_1] [PMID]

[23] Fuortes LJ, Weismann DN, Graeff ML, Bale JF Jr, Tannous $\mathrm{R}$, Peters C. Immune thrombocytopenia and elemental mercury poisoning. Journal of Toxicology: Clinical Toxicology. 1995; 33(5):449-55. [DOI:10.3109/15563659509013753] [PMID]

[24] Kao WF, Hung DZ, Tsai WJ, Lin KP, Deng JF. Podophyllotoxin intoxication: Toxic effect of Bajiaolian in herbal therapeutics. Human \& Experimental Toxicology. 1992; 11(6):4807. [DOI:10.1177/096032719201100607] [PMID]

[25] Afolabi OK, Oyewo EB, Adeleke GE, Badmus JA, Wusu AD. Mitigation of aluminium phosphide-induced hematotoxicity and ovarian oxidative damage in Wistar rats by Hesperidin. American Journal of Biochemistry. 2019; 9(1):7-16. [DOI:10.5923/j.ajb.20190901.03]

[26] Priziola JL, Smythe MA, Dager WE. Drug-induced thrombocytopenia in critically ill patients. Critical Care Medicine. 2010; 38(Suppl 6):S145-54. [DOI:10.1097/ CCM.0b013e3181de0b88] [PMID]

[27] Jones CI. Platelet function and ageing. Mammalian genome. 2016; 27(7-8):358-66. [DOI:10.1007/s00335-016-9629-8] [PMID] [PMCID]

[28] eHealthMe. Will you have Jaundice with methadone hydrochloride? [Internet] [cited: 2020 september 9]; Available from: https://www.ehealthme.com/ds/methadone-hydrochloride/thrombocytopenia/.

[29] Sanidas EA, Tzanis G, Papadopoulos D, Barbetseas J, Papademetriou V. Olmesartan worsening known thrombocytopenia. A rare side effect of antihypertensive drugs. Hellenic Journal of Cardiology. 2017; 58(1):96-7. [DOI:10.1016/j. hjc.2017.01.020] [PMID]

[30] Patel DK, Bilkha N, Schnee D. Angiotensin II type 1 receptor blocker-induced immune thrombocytopenia: A case report. Journal of Medical Case Reports. 2013; 7(1):183. [DOI:10.1155/2013/653925] [PMID] [PMCID]

[31] Patriarcheas V, Pikoulas A, Kostis M, Charpidou A, Dimakakos E. Heparin-induced thrombocytopenia: Pathophysiology, diagnosis and management. Cureus. 2020; 12(3):e7385. [DOI:10.7759/cureus.7385] [PMID] [PMCID]

[32] McKenzie DS, Anuforo J, Morgan J, Neculiseanu E. Successful use of intravenous immunoglobulin $g$ to treat refractory heparin-induced thrombocytopenia with thrombosis complicating peripheral blood stem cell harvest. Journal 
of Investigative Medicine High Impact Case Reports. 2018; 6:2324709618755414. [DOI:10.1177/2324709618755414] [PMID] [PMCID]

[33] Solomon HM, Randall JR, Simmons VL. Heparin-induced increase in the international normalized ratio. Responses of 10 commercial thromboplastin reagents. American Journal of Clinical Pathology. 1995; 103(6):735-9. [DOI:10.1093/ ajcp/103.6.735] [PMID]

[34] Kupeli S. Colistin-related thrombocytopenia. Platelets. 2015; 26(8):812-3. [DOI:10.3109/09537104.2015.1004044] [PMID]

[35] Grim SA, Rene L, Gupta S, Clark NM. Safety of linezolid in patients with baseline thrombocytopenia. Journal of Antimicrobial Chemotherapy. 2008; 62(4):850-1. [DOI:10.1093/jac/ dkn285] [PMID]

[36] Matsumoto K, Takeda Y, Takeshita A, Fukunaga N, Shigemi A, Yaji K, et al. Renal function as a predictor of linezolid-induced thrombocytopenia. International Journal of Antimicrobial Agents. 2009; 33(1):98-9. [DOI:10.1016/j.ijantimicag.2008.07.002] [PMID]

[37] Brier ME, Stalker DJ, Aronoff GR, Batts DH, Ryan KK, O'Grady M, et al. Pharmacokinetics of linezolid in subjects with renal dysfunction. Antimicrobial Agents and Chemotherapy. 2003; 47(9):2775-80. [DOI:10.1128/AAC.47.9.27752780.2003] [PMID] [PMCID]

[38] Watkins VS, Polk RE, Stotka JL. Drug interactions of macrolides: Emphasis on dirithromycin. The Annals of Pharmacotherapy. 1997; 31(3):349-56. [DOI:10.1177/106002809703100 314] [PMID]

[39] Henry-Edwards S, Gowing L, White J, Ali R, Bell J, Brough $R$, et al. Possible drug interactions with methadone: Appendix 1. In: Clinical guidelines and procedures for the use of methadonein the maintenance treatment of opioid dependence. Australian Government Department of Health and Ageing: Publications Production Unit; 2003. file:///C:/Users/Negah-11/Desktop/1512351159_National\%20Guidelines $\% 20$ and $\%$ 20Procedures $\% 20$ for $\% 20$ the $\% 20$ use $\% 20$ of $\% 20$ \%20methadone.pdf

[40] Iribarne C, Berthou F, Baird S, Dréano Y, Picart D, Bail $\mathrm{JP}$, et al. Involvement of cytochrome P450 3A4 enzyme in the N-demethylation of methadone in human liver microsomes. Chemical Research in Toxicology. 1996; 9(2):365-73. [DOI:10.1021/tx950116m] [PMID]

[41] Vandijck DM, Blot SI, De Waele JJ, Hoste EA, Vandewoude KH, Decruyenaere JM. Thrombocytopenia and outcome in critically ill patients with bloodstream infection. Heart \& Lung. 2010; 39(1):21-6. [DOI:10.1016/j.hrtlng.2009.07.005] [PMID] 
This Page Intentionally Left Blank 